\title{
Clinical application and cases examples of a new treatment algorithm for treating thoracic and lumbar spine trauma
}

\author{
Andrei F. Joaquim ${ }^{1}$ - Alpesh A. Patel ${ }^{2}$ - Gregory D. Schroeder ${ }^{3} \cdot$ Alexander R. Vaccaro $^{3}$
}

Received: 24 April 2018 / Revised: 1 June 2018 / Accepted: 2 June 2018

(c) International Spinal Cord Society 2018

\begin{abstract}
Study design Review of illustrative cases of a new algorithm to help in the treatment decision of thoracolumbar spine trauma.

Objectives To illustrate the use of the new algorithm for managing thoracic and lumbar spine trauma.

Settings Recently, a new algorithm for helping in the decision of the best treatment modality for thoracolumbar spine trauma (TLST) was published. The algorithm considers injury morphology, neurological status, clinical status (pain and disability), and also multimodal radiological evaluation (MMRE) in the decision for non-operative versus operative treatment for TLST. Injuries were classified in three groups: (1) stable injuries, (2) potentially unstable injuries/ delayed instability, or (3) clearly unstable injuries.

Methods Cases examples of the algorithm application were presented and discussed.

Results Stable injuries (minor fractures without instability) are non-surgically treated; potentially unstable injuries or associated with delayed instability may be initially managed non-surgically and operative treatment is an option, especially in the setting of important pain, deformity or a new neurological deficit. Clearly unstable injuries are treated surgically as soon as possible to avoid neurological worsening, severe pain, and/ or progressive spinal deformity.

Conclusions Clinical examples of TLST were presented, discussed and classified as stable, potentially unstable and clearly unstable injuries. Further studies addressing the reliability and safety of this algorithm are necessary.
\end{abstract}

\section{Introduction}

Treatment of thoracolumbar spine trauma (TLST) is mainly based on non-operative versus surgical management. This decision is based on many factors, such as injury morphology, neurological, and clinical status and also information obtained with radiological images such as CT scans, MRI, and standing plain radiographs when necessary $[1,2]$.

Andrei F. Joaquim

andjoaquim@yahoo.com

1 Neurosurgery Division, State University of Campinas, Campinas, SP, Brazil

2 Department of Orthopaedic Surgery, Northwestern University Feinberg School of Medicine, Chicago, IL, USA

3 Department of Orthopaedic Surgery and Neurosurgery, Thomas Jefferson University, Philadelphia, PA, USA
Recently, we published a theoretic algorithm grouping injuries in three scenarios, according to the instability definition proposed by Abbasi Fard et al. [3, 4] Injuries were grouped in stable, without risk of neurological deterioration or deformity, treated non-operatively; potentially unstable injuries, who require close radiological and clinical follow-up due to a potential risk of progressive spinal deformity, neurological deterioration and/ or clinical symptoms - surgery may be recommended in this groupand, finally, clearly unstable injuries, who require early surgical treatment due to a higher risk of spinal deformity, severe pain and/ or neurological deterioration [4]. The diagnosis of potentially unstable injuries may also be important to identify patients who may have benefits of additional radiological assessment, such as dynamic radiological exams and MRI, as well as to identify characteristics that may be associated with late surgical treatment.

In this paper, we presented illustrative cases examples to illustrate the clinical application of our proposal algorithm. 


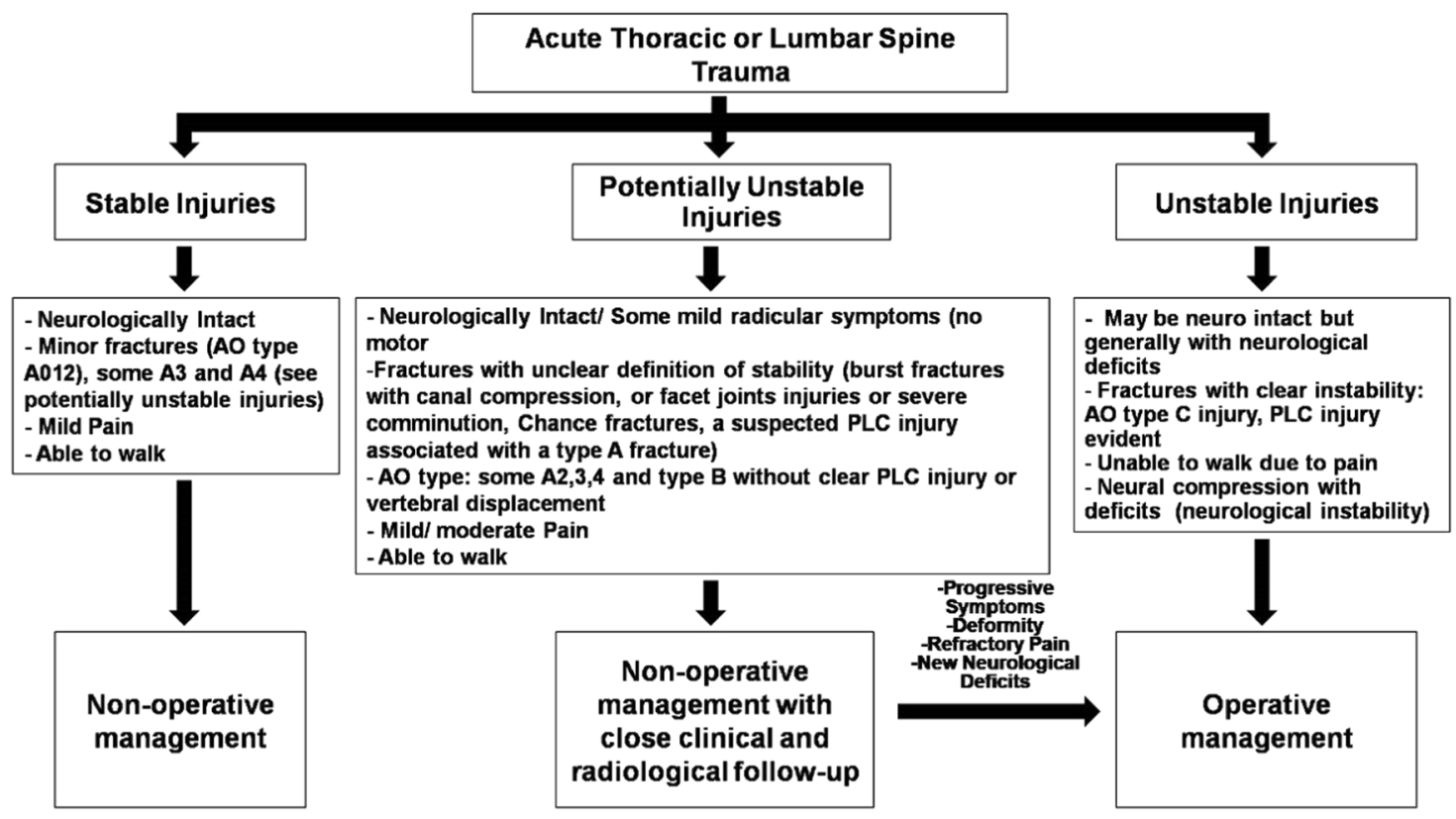

Fig. 1 Flow chart of our suggested treatment algorithm

\section{Methods}

Cases of TLST were retrospectively reviewed from a trauma database (IRB approved-CAAE: 06425012.9.0000.5404). Cases were then classified according to their morphology using the new AOSpine Classifications system, neurological status and clinical status by the authors (Fig. 1-flow Chart of our treatment algorithm) [5]. The new AOSpine classification was recently proposed by Vaccaro et al. for a better morphological categorization, classifying injuries in three main types: type A-compression injuries (compression and burst fractures), type $\mathrm{B}$-distraction injuries and type $\mathrm{C}$ translation injuries (with displacement and dislocation).

The rational of the treatment option was discussed.

\section{Results}

1) Stable injuries-include stable and minor fractures, without neurological deficits and without risk of spinal deformity-characteristics of this group are presented in Table 1.

An illustrative case (Fig. 2) is presented.

2) Potentially unstable injuries-characteristics of this group are presented in Table 2. This group includes borderline injuries whose morphological characteristics are associated with a higher risk of failure of non-operative management.

Illustrative cases (Figs. 3 and 4) are presented.
Table 1 Summary of the main characteristics of stable injuries

\begin{tabular}{ll}
\hline & Stable injuries \\
\hline Morphology & $\begin{array}{l}\text { Minor facet, transverses and spinous process } \\
\text { fractures without any dislocation } \\
\text { Compression fractures, burst fractures with } \\
\text { mild canal compression }\left(<50^{\circ}\right) \text { and without } \\
\text { any associated injury in the facet joints and } \\
\text { without severe vertebral body comminution }\end{array}$ \\
AOSpine & $\begin{array}{l}\text { Some, A3 and A4 subtypes } \\
\text { Classification }\end{array}$ \\
morphologies [5] & $\begin{array}{l}\text { Neurologically intact patients } \\
\text { Neurological status }\end{array}$ \\
Clinical symptoms & $\begin{array}{l}\text { Able to ambulate } \\
\text { CT scan, MRI, and dynamic/ standing plain } \\
\text { Radiological diagnosis }\end{array}$ \\
Treatment & $\begin{array}{l}\text { Non-operative treatment: } \\
\text { - Pain medication }\end{array}$ \\
& - With or without a brace
\end{tabular}

3) Clearly unstable injuries-characteristics of this group are presented in Table 3. These injuries may lead to severe pain, spinal deformity, or neurological worsening when surgical treatment is not performed.

Illustrative cases (Figs. 5 and 6) are presented.

\section{Discussion}

In this paper, we presented a few cases of TLST, with and without neurological deficits, considering our recently 


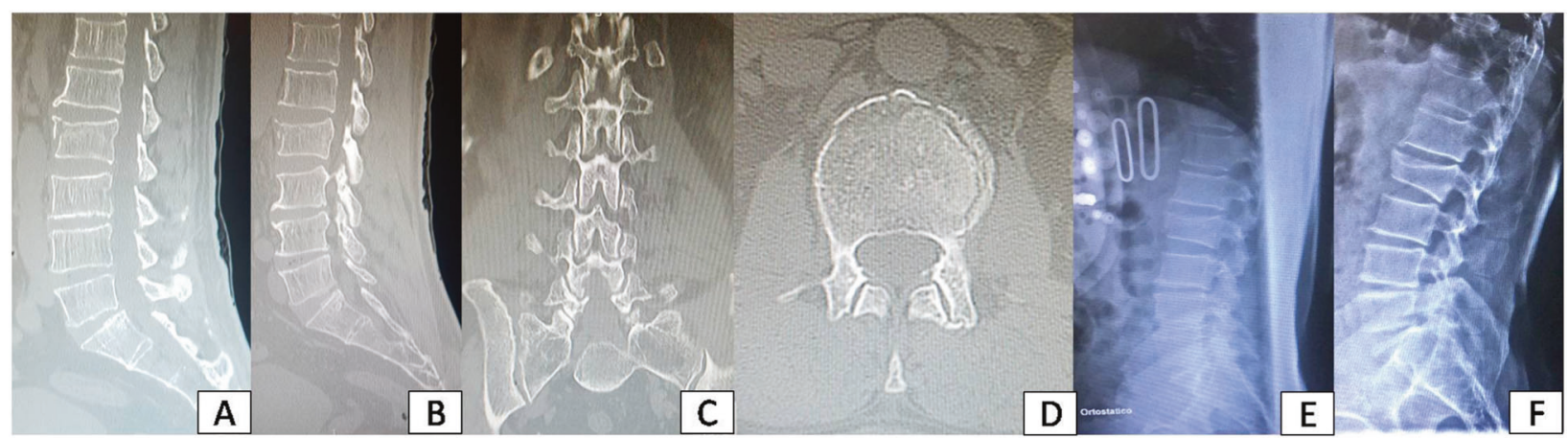

Fig. 2 This 54-year-old man was involved in a bicycle accident with a L2 compression fracture-he was neurologically intact. a Sagittal and b parasagittal CT scan showing a superior endplate L2 compression fracture. c Coronal CT scan without any degree of facet joints displacement and without any fracture evident at the posterior elements. $\mathbf{d}$
Axial CT scan image at L2 with a vertebral body fracture without any degree of canal involvement. e Standing lateral X-ray with a brace without displacement or kyphosis. This injury was considered as stable and non-operative treatment was performed. f Lateral X-ray after 3 months with bone healing. No evident deformity was observed
Table 2 Summary of the main characteristics of potentially unstable injuries

\begin{tabular}{ll}
\hline Potentially unstable injuries \\
\hline Morphologies & Compression/burst fractures with mild increased of the spinous process \\
distance & Burst fractures with moderate or severe canal compression $\left(>50^{\circ}\right)$ \\
& Burst fractures with some diastases of the facet joints but without facet \\
subluxation & Burst fractures with severe comminution \\
Bone chance-type fractures (pedicular fractures without any degree of \\
displacement and without MRI signs of ligamentous injury) \\
Hyperextension fractures with increasing of the disc space without \\
neurological deficits and without displacement of the posterior elements
\end{tabular}

AOSpine classification [5] morphologies

Some A2, A3, and A4 subtypes

B1, B2, and B3 subtypes without displacement

Neurological status

Neurologically intact

Mild radicular symptoms

Clinical symptoms

Mild pain, pain controlled by analgesics

Able to ambulate

Severe and refractory pain may be considered as a sign of instability and early surgical treatment may be considered

Radiological diagnosis

CT scan, MRI, and dynamic/standing plain radiographs

Treatment
Non-operative treatment:

-Pain medication

-Preferentially with a brace

Surgical treatment:

-In the setting of severe worsening of back pain, progressive deformity especially in the setting of worsening of clinical symptoms, new vertebral dislocation, incapacity to walk due to pain and severe clinical impairment after the spine injury proposed treatment algorithm for acute traumatic injuries [4]. The idea is that, considering not only CT scan and fracture morphology, but also other image modalities, such as standing plain radiographs and MRI, we can improve the way we treat TLST based on additional information, such as ligamentous status and load effect into the vertebrae. On the other hand, although many fracture patterns are quite similar, such as a burst fracture in the thoracolumbar junction, the details of each case are unique, such as pain perception, neurological deficits (radicular, incomplete, and complete) and fracture characteristics (such as degree of canal compromise, vertebral body comminution, posterior elements fractures, among others).

Historically, many studies addressed radiological characteristics of spinal fractures in an attempt to decide the best treatment modality. Some of these characteristics include the degree of canal compression, local kyphosis $\left(>20-30^{\circ}\right)$, and vertebral body comminution (such as the load sharing 


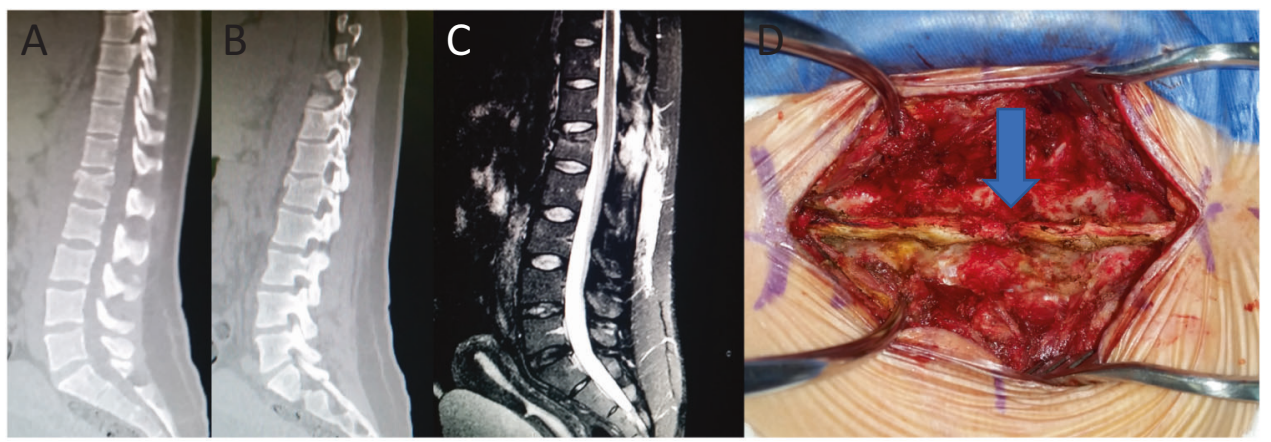

Fig. 3 This 34-year-old woman fall from the height and was neurologically intact. She had an initial bipedicular fracture at L1 classified as B1, as shown in $\mathbf{a}$ and $\mathbf{b}$ sagittal CT scan. Due to inability to stand, and a concern for a possible ligamentous injury as evident by spinous process splaying, an MRI was performed. c This demonstrated a suspect posterior ligamentous complex (PLC) injury, as shown in the sagittal T2 MRI. We classified this injury as potentially unstable and surgery was offered once she was not able to stand without significant pain even using a brace and after pain medication. d Intraoperatively picture showing disruption of the PLC (blue arrow). The patient was able to walk two days after surgery

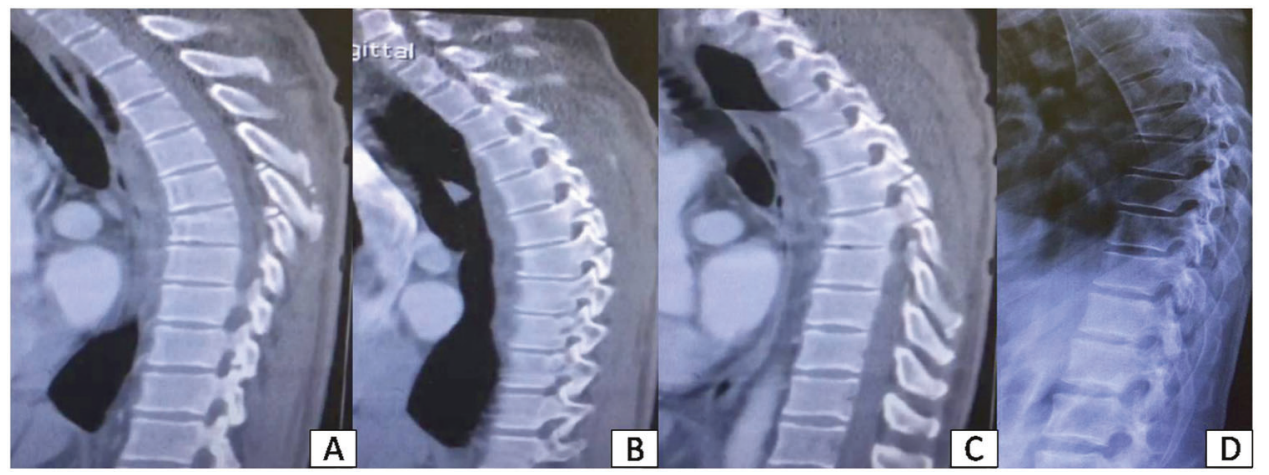

Fig. 4 This 42-year-old man who fell from a significant height while working. He was neurologically intact. a Sagittal CT scan with an A1 fracture at T7, with spinous process fracture at T6 and T7. b, c Left and right parasagittal CT scan with bilateral facet fractures as well. There was no dislocation although the fracture was in the apex of the thoracic kyphosis. A potential posterior ligamentous complex injury was inferred but not clearly diagnosed. $\mathbf{d}$ A standing lateral thoracic Xray was performed without any evident degree of displacement and no additional pain. The fracture was likely stable, and so initial nonoperative care was attempted; importantly, close clinical, and radiological follow-up was needed to ensure there was no late instability
Table 3 Summary of the main characteristics of potentially unstable injuries

\begin{tabular}{|c|c|}
\hline & Clearly unstable injuries \\
\hline Morphologies & $\begin{array}{l}\text { Distractive or rotational injuries, with vertebral dislocation, spinal } \\
\text { misalignment, facet subluxation or luxation, total canal occlusion, clear } \\
\text { and evident increasing in the distance of the spinous processes. }\end{array}$ \\
\hline $\begin{array}{l}\text { AOSpine Classification } \\
\text { morphologies [5] }\end{array}$ & $\begin{array}{l}\text { All injuries with a significant neurologic deficit } \\
\text { All Type C injuries } \\
\text { Some A3 and A4 injuries; some B1-3 injuries }\end{array}$ \\
\hline Neurological status & $\begin{array}{l}\text { Neurologically intact or } \\
\text { Complete or incomplete neurological deficits (exception: splashing } \\
\text { injuries without compression) } \\
\text { Moderate or severe radicular symptoms specially those refractory to pain } \\
\text { medication }\end{array}$ \\
\hline Clinical symptoms & Patients may have mild to severe pain, considering injury morphology \\
\hline Radiological diagnosis & $\begin{array}{l}\text { CT scan. MRI may be necessary to evaluate soft tissue compression or } \\
\text { neurological deficits not explained by CT scan findings }\end{array}$ \\
\hline Treatment & $\begin{array}{l}\text { Early surgical fixation is recommended for stabilize the spine and, when } \\
\text { necessary, realigned it and decompress the neural tissue }\end{array}$ \\
\hline Follow-up & Regular clinical and radiological follow-up \\
\hline
\end{tabular}




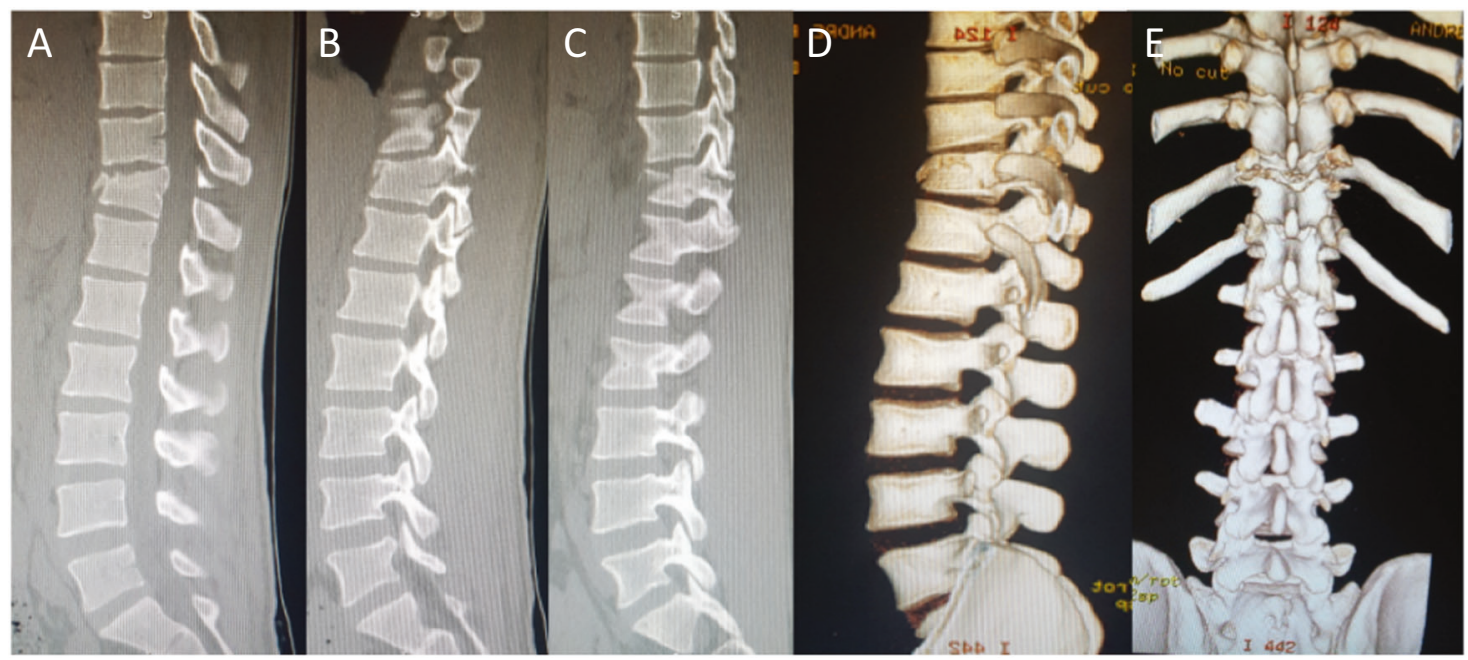

Fig. 5 This 26-year-old man was involved in a car accident with a T11 fracture-he was neurologically intact. a Sagittal and b, c parasagittal CT scan showing superior endplate fracture at T11 with bilateral pedicular fracture at T11. In a and in the 3D CT reconstruction $(\mathbf{d}, \mathbf{e})$, we can see some important increasing in the distance of the spinous process indicating a posterior ligamentous lomplex injury at T10 and T11 and surgical treatment was performed

Fig. 6 This 43-year-old man had a motorcycle accident with severe radicular pain and mild leg weakness (AIS D). He was also unable to sit. He had a burst fracture at $\mathrm{L} 4$, as seen in the a sagittal $\mathbf{C T}$ and $\mathbf{b}$ axial $\mathrm{CT}$, with important canal compression. c Axial and d sagittal T2 sequence MRI with radicular compression. This injury was indicated for surgery secondary to the radiculopathy and leg weakness, with neurological risk of deterioration (neurological instability) with a posterior surgical approach. The patient was AIS E at 6 months of follow-up with minor pain
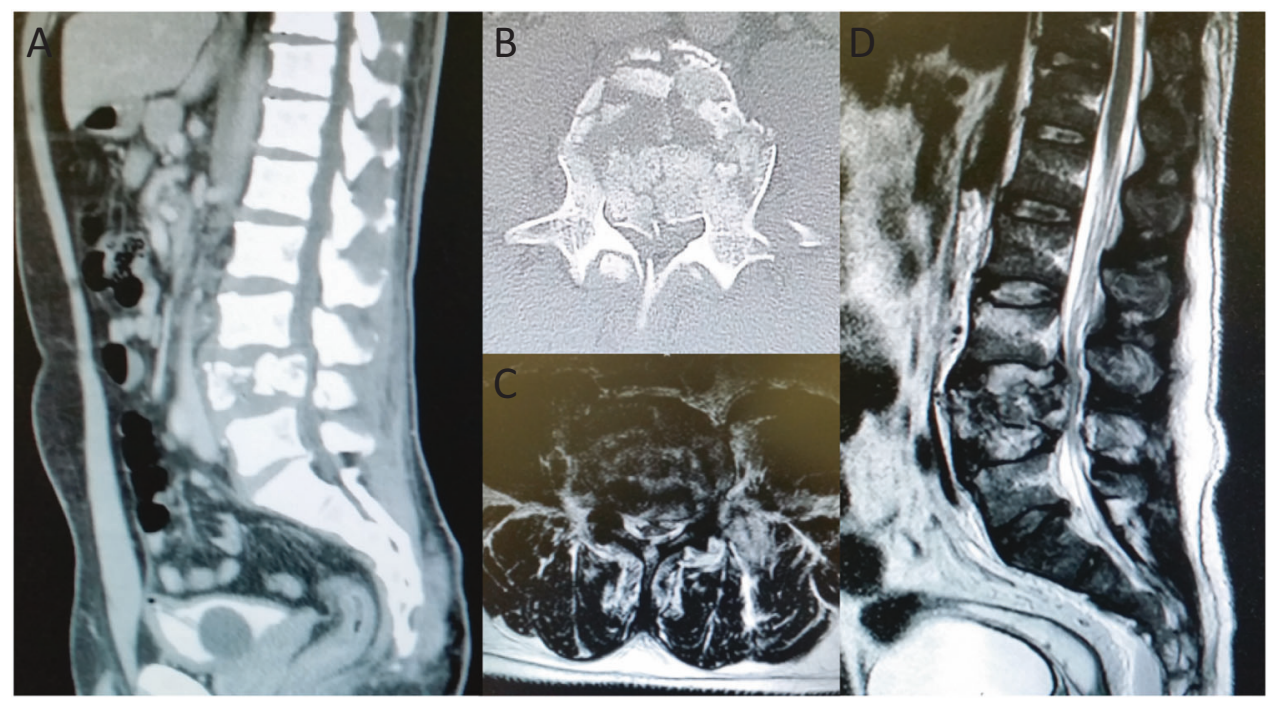

classification system) [6-10]. Interesting, normal thresholds for surgical versus non-operative management are difficult to be defined. As an example, normal wedging of a vertebral body (the ratio between the anterior and posterior vertebral height) in asymptomatic adults has been suggested to be up to $10^{\circ}$, although some authors reported up to $25^{\circ}$ as a normal wedge limit [11-15]. This variation of what is considered normal may result in potentially different outcomes and interpretation of a compression fracture in an adult patient, especially in the setting of degenerative spine disease. By this reason, we think that a large group of spinal injuries, with "borderline" morphological characteristics, may be better grouped as "potentially unstable" injuries, which are at higher risk of failure non-operative management. For security, this group did not include patients with spinal dislocation, where ligamentous injury was clearly evident and surgical treatment is recommended, even in patients without neurological deficits $[15,16]$.

Radcliff et al. evaluated prospectively 46 patients with traumatic burst fractures from T10-L2. The objective of the study was to assess if the degree of canal compromise (CC) and neurological deficits correlate with posterior ligamentous injury (PLC) and neurological deficits [17]. Loss of vertebral body height (LOVBH), vertebral translation, local kyphosis (LK), and CC were evaluated, as well as ligamentous and neurological injury. Ligamentous injury was assessed based on the PLC on MRI signal of the supraspinous ligament, ligament flavum and interspinous ligament. Ten patients had kyphosis $>20^{\circ}$ and one patient had $>30^{\circ}$. Nine patients had $>50 \%$ of LOVBH. Vertebral 
body translation $>3.5 \mathrm{~mm}$ was documented in 34 patients and $50 \%$ of the patients had CC $>50 \%$. PLC injury was detected in 16 cases. Segment translation $>3.5 \mathrm{~mm}$ strongly correlates with ligamentous injury $(p=0.029)$ and ASIA motor score $(p=0.009)$. PLC injury and neurological status did not correlate with LOVBH $>50 \%$, vertebral body kyphosis $>20^{\circ}$, caudal or cephalad interspinous widening $>7 \mathrm{~mm}$ or $\mathrm{CC}>50 \%$. They concluded that the LOVBH and LK did not correlate with PLC injury in burst fractures at the thoracolumbar junction. On the other hand, segment translation correlates with the integrity of the PLC. A potential explanation of this is that severe bone destruction may expect to have less ligamentous injury because the injury impact was more evident on the bone than in the soft tissues. However, the role of PLC injury in the outcome of TL burst was not evaluated.

These individual radiological characteristics should also be considered together with the complex trauma scenario and in the presence of other comorbidities. A patient with a potentially unstable injury but who will be lying down for some weeks, for instance, due to a severe traumatic injury, may be considered for non-operative treatment, whereas the same fracture in a young and healthy adult may require surgical fixation. Conversely, early surgery may be given to the poly-trauma patient if bracing is not possible secondary to other injuries.

To maintain long-term spinal stability and also neurological preservation is the main treatment goal in patients without neurological deficits, whereas in patients with neurological impairment, early decompression and spinal stabilization are the main objectives $[1,2]$.

Stable injuries are generally associated with good clinical outcomes, once they generally heal well $[1,2]$. On the other hand, injuries that may develop spinal deformity or chronic pain are more complex, once the role of surgical intervention is uncertain. Finally, unstable injuries may result in spine deformity due to loss of the ability to sustain the body weight under physiological loads. On the same context, a spine trauma may be considered "unstable" from a neurological point of view in the setting of neurological deficits and persistent neural tissue compression. In this situation, surgical treatment may be recommended [2].

The potential advantage of our algorithm is to classify some injuries as potentially unstable, which may benefit from additional radiological evaluation with MRI and dynamic or standing/load plain radiographs for further information, as well as considering some clinical data, such as mechanical and persistent pain, before treatment decision. Patients with potentially unstable injuries may also be selected for future clinical trials to evaluate the role of surgical versus non-operative treatment in their long-term outcome.

\section{Conclusions}

The rational of treatment of TLST was explained based on multiple factors, such as radiological characteristics, patients' symptoms, and neurological status. The best treatment option is discussed with some case examples. Prospective clinical studies are necessary to evaluate the best treatment modality for some specific fracture patterns, mostly those included in our group of potentially unstable injuries.

\section{Compliance with ethical standards}

Conflict of interest The authors declare that they have no conflict of interest.

\section{References}

1. Vaccaro AR, Zeiller SC, Hulbert RJ, Anderson Pa, Harris M, Hedlund R, et al. The thoracolumbar injury severity score: a proposed treatment algorithm. J Spinal Disord Tech. 2005;18:209-15.

2. Joaquim AF, Patel AA. Thoracolumbar spine trauma: evaluation and surgical decision making. J Craniovert Jun Spine. 2013;4:3-9.

3. Abbasi Fard S, Skoch J, Avila MJ, Patel AS, Sattarov KV, Walter $\mathrm{CM}$, Baaj AA. Instability in thoracolumbar trauma: is a new definition warranted? Clin Spine Surg. 2017;30:E1046-1049.

4. Joaquim AF, Patel AA, Schroeder GD, Vaccaro AR. A simplified treatment algorithm for treating thoracic and lumbar spine trauma. J Spinal Cord Med. 2018;7:1-11.

5. Vaccaro AR, Oner C, Kepler CK, Dvorak M, Schnake K, Bellabarba C, et al. AOSpine Spinal Cord Injury \& Trauma Knowledge Forum. AOSpine thoracolumbar spine injury classification system: fracture description, neurological status, and key modifiers. Spine. 2013;38:2028-37.

6. Munting E. Surgical treatment of post-traumatic kyphosis in the thoracolumbar spine: indications and technical aspects. Eur Spine J. 2010;19:69-73.

7. McCormack T, Karaikovic E, Gaines RW. The load sharing classification of spine fractures. Spine. 1994;19:1741-4.

8. Cantor JB, Lebwohl NH, Garvey T, Eismont FJ. Non-operative management of stable thoracolumbar burst fractures with early ambulation and bracing. Spine. 1993;18:971-6.

9. McAfee PC, Bohlman HH, Yuan HA. The value of computed tomography in thoracolumbar fractures: An analysis of one hundred consecutive cases and a new classification. J Bone Jt Surg Am. 1985;67:89-104.

10. Gaca AM, Barnhart HX, Bisset GS 3rd. Evaluation of wedging of lower thoracic and upper lumbar vertebral bodies in the pediatric population. AJR Am J Roentgenol. 2010;194:516-20.

11. Genant HK, Wu CY, van Kuijk C, Nevitt MC. Vertebral fracture assessment using a semiquantitative technique. J Bone Miner Res. 1993;8:1137-48.

12. Szulc P, Munoz F, Marchand F, Delmas PD. Semiquantitative evaluation of prevalent vertebral deformities in men and their relationship with osteoporosis: the MINOS Study. Osteoporos Int. 2001;12:302-10.

13. McKiernan FE. The broadening spectrum of osteoporotic vertebral fracture. Skelet Radiol. 2009;38:303-8.

14. Matsumoto M, Okada E, Kaneko Y, Ichihara D, Watanabe K, Chiba K, Toyama Y, Fujiwara H, Momoshima S, Nishiwaki Y, 
Hashimoto T, Takahata T. Wedging of vertebral bodies at the thoracolumbar junction in asymptomatic healthy subjects on magnetic resonance imaging. Surg Radiol Anat. 2011;33:223-28.

15. Haiyun Y, Rui G, Shucai D, Zhanhua J, Xiaolin Z, Xin L, et al. Three-column reconstruction through single posterior approach for the treatment of unstable thoracolumbar fracture. Spine. 2010;35:E295-302.

16. Wang XB, Yang M, Li J, Xiong GZ, Lu C, Lu GH. Thoracolumbar fracture dislocations treated by posterior reduction interbody fusion and segmental instrumentation. Indian J Orthop. 2014;48:568-73.

17. Radcliff K, Su BW, Kepler CK, Rubin T, Shimer AL, Rihn JA, Harrop JA, Albert TJ, Vaccaro AR. Correlation of posterior ligamentous complex injury and neurological injury to loss of vertebral body height, kyphosis, and canal compromise. Spine. 2012;37:1142-50. 